\title{
Sexualidad y afecto entre los macuna y los nükak, pueblos de la amazonia colombiana*
}

\author{
Dany Mahecha Rubio**
}

En este texto exploro cómo los indígenas de la Amazonia colombiana vivencian y expresan la sexualidad y el afecto. Argumento que estos asuntos están estrechamente relacionados con una noción de persona construida a partir de la pertenencia a un cuerpo social y de una concepción del cuerpo que marca las diferencias de género para unas cosas, pero que las matiza para otras. Para desarrollar este argumento me baso principalmente en información etnográfica de los macuna ${ }^{1}$ y los nükak ${ }^{2}$, dos pueblos cuyos modos de vida presentan elementos similares y otros notablemente diferentes. ${ }^{3}$ Así, mientras que los macuna son

\footnotetext{
* Recebido para publicação em 20 de setembro de 2013, aceito em 30 de outubro de 2013.

** Docente de las Universidad Nacional de Colombia UNAL Sede Amazonia. dmahecharu@unal.edu.co

1 Los macuna habitan principalmente en el Pirá-Paraná y el bajo Apaporis. Su lengua, con 750 hablantes aproximadamente, pertenece a la familia Tucano oriental.

2 Los nükak habitan el interfluvio Guaviare-alto Inírida. Su lengua cuenta con 650 hablantes y su clasificación como parte de la familia Makú-Puinave está en discusión, sin resultados conclusivos (Mahecha, 2007).

3 La información en la que se fundamenta este ensayo proviene de trabajos de campo realizados con: 1) los macuna del Bajo Apaporis, entre el 2001 y el 2003, como parte del desarrollo de la tesis de Maestría en Estudios Amazónicos de la Universidad Nacional de Colombia Sede Amazonia (UNAL); y 2) con los nükak, relacionados con la elaboración de la tesis de investigación en Antropología de la UNAL Sede Bogotá, entre 1991-1993, los programas de investigación con pueblos nómadas desarrollados por la Fundación Gaia Amazonas entre 1995, 1997, y los llevados a cabo durante la elaboración de la tesis doctoral en Letras de la Vrije Universiteit, Ámsterdam 2005-2013, en curso. También quiero agradecer a Carlos Franky por el entusiasmo en las discusiones y comentarios a la versión preliminar de este texto.
}

cadernos pagu (41), julho-dezembro de 2013:63-75. 
Sexualidad y afecto entre los macuna y los nükak...

horticultores que están interactuando con europeos y criollos desde el siglo XVIII (véase Mahecha 2004, Cayón 2010), los nükak son cazadores y recolectores nómadas que permanecieron en aislamiento hasta la década de los 1970, afrontando desde entonces un proceso de contacto que los ha llevado a una situación de alta vulnerabilidad (Cabrera et al, 1999; Franky, 2011; y Mahecha et al, 2010). Sin embargo, ambos pueblos comparten aspectos como la filiación patrilineal, la virilocalidad, la poligamia, la prescripción matrimonial entre primos cruzados y una sexualidad ligada fundamentalmente a la reproducción humana.

Empezaré por señalar que entre los macuna y los nükak la noción de persona está imbricada con la construcción del cuerpo. Esta se concibe como el resultado del trabajo individual $y$ colectivo, especialmente de los parientes con quienes se reside a lo largo de la vida. Desde que nacen, los cuerpos de los niños son involucrados en un proceso que los feminiza o masculiniza de acuerdo con los dominios de conocimiento y acción de cada género, en función de las expectativas de la colectividad. A su vez, la colectividad está involucrada en una red de relaciones con otros seres humanos y espirituales, que se perciben como consanguíneos o afines, para garantizar el bienestar físico y espiritual de todos los seres del universo (Cfr. Århem, 2001; Cayón, 2002; Franky, 2011; Mahecha, 2004; Mahecha et al, 2013).

Las cosmologías amazónicas afirman que una parte de las diversas clases de seres del cosmos comparten el estatus ontológico de "gente", reconociendo entre ellas una condición de humanidad genérica primordial común, sin importar el nivel o mundo del cosmos donde habiten (Cfr. Århem, s.f; Viveiros de Castro, 2002). Sin embargo, para diferenciarse de las otras gentes, cada pueblo indígena marca su especificidad valorándose así mismo como "la gente verdadera", lo cual expresan tácita o explícitamente en su autodenominación. Pero esta diferenciación es una potencialidad a desarrollar, en un proceso de construcción particular del cuerpo de sus hijos y demás descendientes en el que se le imprimen las características específicas de "su propia gente". 
Así desde las primeras horas de vida, a un neonato se lo empieza a transformar en un ser "plenamente" humano, a través del contacto con substancias "propias" de la gente verdadera (pintura y adornos corporales, leche materna, curaciones chamanísticas, etc.). Si esto no se hiciese, el neonato podría "morir"; es decir, otras gentes del cosmos se lo llevarían para criarlo o devorarlo (Cfr. Cayón 2010; Franky 2011; Mahecha, 2004) ${ }^{4}$

Es desde esta percepción de la alteridad que se erige la noción de persona, y así como cada clase de gente requiere marcar su especificidad, la creación de personas femeninas y masculinas también demanda fijar las diferencias entre los géneros y al mismo tiempo reconocer la necesidad de su complementariedad para reproducirse como individuos y como cuerpo social. Para muchos pueblos de filiación patrilineal, la sangre femenina y el semen masculino son las primeras substancias constitutivas de un ser humano, pero la fecundación solo es factible a través de coito múltiple - con uno o varios hombres ${ }^{5}$, ya que el embrión se alimenta y crece con el semen hasta que se desarrolla y se alimenta por el cordón umbilical. Es así como la sangre y semen de los padres están impregnados de las substancias alimenticias y rituales que manejan "la gente" a los que pertenecen tanto por su respectiva filiación patrilineal como por el género (Cayón, 2010, Franky, 2011, Mahecha, 2004).

${ }^{4}$ Las etnografías de otros pueblos amazónicos revelan que la gestación y nacimiento de un individuo también requiere, además de una preparación adecuada de los padres, de curaciones chamanísticas en las que participan espíritus aliados consanguíneos de la colectividad, o en donde los neonatos deben ser presentados a aquellos seres que residen en su territorio y al mismo tiempo protegidos de los potenciales que peligros que otros seres representan, véase para los tanimuca Franky (2004) y para los wari Vilaça (2002).

${ }_{5}$ Actualmente entre los macuna la práctica del coito múltiple tiende a negarse, pero su permanencia, como práctica cultural, se evidencia en las bromas cotidianas sobre el tema; y en la importancia que le dan a este aspecto en las curaciones chamánicas. En contraste, para los nükak el coito múltiple con varios hombres, durante la gestación, le permite al embrión tomar algunos rasgos de la personalidad de estos a través del semen. 
Sexualidad y afecto entre los macuna y los nükak...

Para los macuna, los cuerpos femeninos están hechos principalmente de yuca brava, ají y tubérculos y frutas cultivadas; mientras que los masculinos lo están de coca, tabaco y yagé. Si bien asumen que la consubstancialidad que se produce entre estas plantas y las personas que las manejan contribuye a apropiar las características de cada género, los "cuerpos espirituales" de las mujeres también se forman con coca y tabaco y los de los hombres con yuca brava y otras especies cultivadas (Cayón, 2002, 2010; Mahecha, 2004). Esto debido a que estas substancias también constituyen, protegen y defienden los cuerpos del otro género. En contraste, para los nükak los cuerpos femeninos y masculinos están constituidos por las mismas substancias, especialmente por los jugos de palmas y frutales comestibles, pero los hombres son los únicos que pueden manejar el eoro, una substancia usada con fines chamanísticos hecha de una planta tintórea con la intervención de espíritus aliados.

Durante el proceso de socialización, una de las primeras y principales enseñanzas a un niño es que aprenda a cuidar su cuerpo. Dichas enseñanzas se transmiten a través de los consejos morales, la alimentación adecuada y las demás prácticas cotidianas, chamanísticas y rituales. Con ello se busca "endurecer" el cuerpo, preparándolo para manifestar plenamente en la adultez capacidades reproductivas "biológicas" y "sociales" en la procreación y crianza, la obtención de alimentos, la consolidación de alianzas o en el chamanismo y el ritual. Paralelamente, se le enseña a controlar comportamientos y emociones, y a manejar apropiadamente las relaciones afectivas y la sexualidad. En otras palabras, a "pensar bien", como dicen los macuna (Mahecha, 2004), o a "estar contentos y acompañados" como señalan los nükak (Franky, 2011).

Esta filosofía del aprendizaje enfatiza y estimula la adquisición de un alto sentido de la autonomía, que les permita satisfacer las demandas de los cónyuges - compañía, afecto, sexo, bienes, alimentos, protección y conocimientos -, las de los hijos y otros parientes coresidentes, consanguíneos y afines, con los que 
efectúan múltiples labores cotidianas y con quienes también comparten cuidados y substancias alimenticias y rituales. ${ }^{6}$ Así, las manifestaciones de afecto más reiterativas se expresan en los cuidados prodigados en la relación entre criadores y criados. Los hijos son la alegría de una casa, pero también la posibilidad de pervivir como individuo y como "gente" en el futuro, por ello para los macuna los hijos son "el reemplazo de uno" (Mahecha, 2004).

Para los macuna una persona como yo, que aunque tenía esposo no tenia hijos, hasta hace unos años, era considerada como "mezquina" (egoísta) por no dar hijos a mi cónyuge, "perezosa" por no trabajar con suficiente ahínco para embarazarme y procrear, y como una mujer con un problema de fertilidad, porque alguien había invertido mi 'banco de pensamiento' de la fertilidad (el útero). Los nükak por su parte me curaron chamanísticamente un par de veces para propiciar mi embarazo. Cuando se confirmó la gestación, me indicaron que mi bebé debía llamar a Kerayi, el anciano que me curó, abuelo y a su hijo Dugupbe, quien también colaboró, padre (asumido como un hermano consanguíneo de mi esposo). Por ende nosotros debíamos asumir una serie de obligaciones como parientes, ya que desde la perspectiva nükak ellos le dieron la vida a nuestra hija.

Lo anterior sugiere que el propósito más relevante de la sexualidad es asociado a la reproducción humana ${ }^{7}$, y percibido como un asunto que le compete no solo a los conyugues sino a un cuerpo social. Sin embargo, aunque los macuna y los nükak consideran que tener sexo es agradable y fuente de placer, no es la prioridad en la cotidianidad de un adulto con una unión conyugal estable y con hijos. Además durante diversos momentos de la vida, como la reclusión posparto, las reclusiones menstruales

6 La autonomía como parte de la formación de la persona es un rasgo común en diversos pueblos de la Amazonia, como los airo pai (Belaunde, 2001), los piro (Gow, 2001), los nonuya Londoño (2005), los piaroa (Overing et al, 2000) y los huaorani (Rival, 1996, 1998).

7 Al igual que para los mehinaku (Gregor, 1985) y los huaorani (Cfr. Rival). 
Sexualidad y afecto entre los macuna y los nükak...

de las mujeres, la participación en rituales masculinos, algunos bailes y durante ciertas enfermedades, tener sexo atenta contra el propio bienestar y el de otras personas. Por ello la separación de los cuerpos evita que las substancias masculinas y femeninas se mezclen inapropiadamente, pues así como unas substancias son fuentes de poder, conocimiento y fertilidad para un género (la sangre menstrual para las mujeres o el eoro para los hombres nükak), también son fuente de peligros para el otro. Es por esta razón que cuando las mujeres cesan de menstruar, pueden consumir algunas substancias masculinas e incrementar el manejo de conocimientos chamanísticos.

Paradójicamente, el discurso macuna y nükak que exhorta a tener sexo para procrear, contrasta con las frecuentes acusaciones sobre adulterio. En general, los adulterios cometidos por los hombres son más tolerados y los de las mujeres más cuestionados y castigados. Desde otra perspectiva, los adulterios entre afines, coresidentes o visitantes, también son más tolerados que entre consanguíneos o agnados, los cuales son severamente cuestionados por su carácter incestuoso. Las recriminaciones para las parejas involucradas se intensifican si se llega a presentar un embarazo. En otras épocas se recurría al infanticidio, pero cada vez es más frecuente el madre solterismo (Mahecha, 2004).

Para los hombres macuna adultos un encuentro sexual furtivo es percibido como una manera de "descargarse" o de "botar" el semen. ${ }^{8}$ Es una especie de acto fisiológico más y no precisamente un encuentro apasionado, erótico o romántico. Para explicar algunas de estas actuaciones, que se volvían un asunto público, los hombres bromeaban con la expresión "todos necesitamos". Por su parte, las mujeres oscilan entre admitir tales encuentros como una picardía intencionada y provocada sin mayores consecuencias, o como situaciones en las que, en contra de su voluntad, tuvieron que acceder a los caprichos de uno o varios hombres. Los conflictos serios por adulterio no son

${ }^{8}$ Datos no publicados de Carlos Franky. 
frecuentes y sólo se dan cuando se compromete la estabilidad de la unión conyugal. Estos pueden implicar fuertes discusiones públicas, agresiones físicas contra la mujer o la separación temporal de la pareja. Las mujeres y los hombres son conscientes de estos peligros y por tanto procuran que estos encuentros sean secretos o discretos.

Los macuna y los nükak no le dan la mayor importancia a la virginidad como criterio para establecer una unión conyugal. Incluso es común que cada uno de los futuros conyugues conozca una parte de quiénes han sido los compañeros sexuales del otro, pues en muchos casos las parejas han tenido algún tipo de acercamiento, que no siempre se puede equipar a un noviazgo. Además, desde la adolescencia los jóvenes tienen una vida sexual activa, la cual si bien no es estimulada por los adultos, tampoco es condenada. Estos más bien tratan de orientarla a través de discursos como el control de las emociones, el manejo de la fertilidad o la prohibición del incesto. En general, establecer una unión matrimonial genera tensiones, pero estas sólo se transforman en conflictos cuando se dan sin el consentimiento de los padres o parientes mayores, ya que desde la infancia se conoce quiénes pueden ser las parejas potenciales de una persona, debido a que el matrimonio se enmarca en las relaciones de alianza entre grupos afines (Cfr. Mahecha, 2004, Franky, 2004; 2011).

Sin bien contemporáneamente los macuna y los nükak admiten una mayor injerencia de una persona en la selección de su futuro conyugue, en el pasado los padres macuna tenían mayor control sobre esta decisión que los nükak (Cfr. Århem, 1989). Así los y las jóvenes nükak contemporáneos han gozado de mayor libertad tanto para escoger y convivir con una pareja como para separarse asumiendo esta decisión unilateral o conjuntamente. En todo caso una unión marital sólo se considera estable luego del nacimiento del primer hijo. Las mayores tensiones que actualmente se presentan en torno a una unión marital se deben al incremento de uniones entre mujeres nükak y hombres no-nükak, pues esto implica la pérdida de esposas potenciales y por tanto de 
Sexualidad y afecto entre los macuna y los nükak...

la reproducción social y de la alianza matrimonial (Mahecha et al, 2010; Franky, 2011).

Entre los macuna las relaciones entre hombres y mujeres afines son más formales y distantes que entre personas del mismo género, las cuales son caracterizadas por la solidaridad y la camaradería, siendo un tema recurrente los chistes con connotaciones sexuales, sobre el comportamiento de sus cónyuges $\mathrm{u}$ otras personas. A su vez, la homosexualidad masculina se reprueba, porque es inconcebible un hombre que no pueda procrear, y por ende en casos de infertilidad siempre se pone en duda las capacidades de las mujeres y nunca las de los hombres.

Los jóvenes son los más dados a trasgredir los protocolos de los mayores respecto a las relaciones heterosexuales en la separación de espacios femeninos y masculinos y a desafiar la normas prescriptivas del matrimonio. Asunto que los mayores atribuyen a la vida en las comunidades, donde se tiene mayor posibilidad de relacionarse con personas de la misma edad y a la escuela, donde son comunes los enamoramientos, las cartas con declaraciones y canciones de amor, situaciones que en general han contribuido a cuestionar los matrimonios arreglados por los mayores. Las parejas más jóvenes suelen reposar en la misma hamaca y tomar los baños cotidianos juntos. Mientras aquellas que llevan más años de vida marital son más recatadas en las expresiones de afecto en público y aunque se asume que el lugar ideal para los encuentros sexuales son las zonas con cultivos, también se dan en la intimidad del hogar de las parejas que viven en casas unifamiliares.

Entre los nükak las relaciones entre consanguíneos y afines residentes es menos tensa, y las bromas con insinuaciones sexuales son recurrentes de parte de los hombres hacia las mujeres solteras. En general, después de la menarquia se asume que las jóvenes ya pueden tener sexo. Y en efecto ellas son "asediadas" por los jóvenes para que les dejen compartir la hamaca o les reciban alimentos que ellos llevan y los preparen, pues estas son señales afirmativas del interés en establecer una unión con ellos. 
Las parejas jóvenes pasan parte de su tiempo retozando en las hamacas en medio de abrazos y mordiscos en público. Aunque en general enuncian que prefieren tener sexo en el bosque, también suelen hacerlo discretamente en el campamento, mientras los demás duermen.

Las manifestaciones de afecto como abrazos o compartir una hamaca entre hombres jóvenes y aun adultos, así como los chistes $e$ insinuaciones sexuales en la que se tocan los genitales son parte de la cotidianidad y son concebidas como asuntos normales, e incluso es factible que entre los jóvenes tengan sexo homosexual, en esta etapa de la vida, pero estas cesan una vez que hayan establecido una unión conyugal. Entre las mujeres los abrazos y la colaboración mutua para peluquearse, pintar sus rostros o desparasitar los cuerpos después de una jornada en el bosque, es un asunto cotidiano con el que expresan los afectos en una complicidad sin igual.

Entre los macuna las prácticas sexuales consideradas más peligrosas son aquellas que se dan entre humanos verdaderos $y$ seres que no son humanos verdaderos, que habitan en otras esferas del cosmos. En cuanto tener sexo o consumir alimentos y substancias con éstos seres puede afectar la salud de la persona, trasformar su propia corporalidad, asumirlos como parientes $y$ desear residir con ellos. ${ }^{9}$ En el caso de los nükak las fronteras de estas relaciones son más flexibles ya que los hombres con conocimientos chamanísticos pueden tener relaciones con mujeres de otros mundos, $e$ incluso las consideran esposas, pero siempre que las visitan deben estar acompañados de espíritus aliados y limitar el consumo de alimentos que les brindan, ya que de aceptarlos todos podrían abandonar a los parientes en este mundo (Franky, 2011).

9 Otros pueblos amazónicos como los mehinaku (Cfr. Gregor, 1985) y los huaorani (Cfr. Rival, 2007) comparten esta percepción. 
Sexualidad y afecto entre los macuna y los nükak...

Consideraciones finales

He tratado de mostrar que para comprender la sexualidad y el afecto macuna y nükak es necesario enmarcarlos en sus respectivas nociones de persona, pues estas enfatizan en la construcción de un cuerpo individual y colectivo específico. Es por ello que el principal propósito de la sexualidad es la reproducción/perpetuación de los padres y sus "gentes". En este sentido se valora altamente la fertilidad femenina y la capacidad de las personas de cuidar/criar a otras. Estos y otros elementos son vistos como esenciales para considerar a alguien como "verdaderamente humano". Es claro que estas son construcciones de la sexualidad y el afecto situadas en un contexto cultural, político e histórico, y con criterios particulares respecto a la corporalidad, la moralidad, las emociones y los deseos. Como también lo es la perspectiva occidental hegemónica heterosexual, la cual contrasta con las anteriores al estar más centrada en un cuerpo biofísico individual universal y una sexualidad altamente erotizada (Cfr. Foucault, 1977; Rubin, 1986; y Weeks, 1995).

Sin embargo, esto no niega ni omite que estas construcciones se hayan estado retroalimentando y trasformado, con experiencias interculturales muchas veces conflictivas en un "diálogo de sordos". En este sentido, diferentes aspectos de estas interacciones requieren una mayor documentación. Para finalizar, quisiera resaltar dos de las posibles líneas de investigación. La primera tiene que ver con la historia. Por ejemplo, la principal diferencia en la manera como expresan los afectos y la sexualidad los macuna y los nükak está dada en términos de las relaciones cotidianas entre consangüíneos y afines coresidentes. Entre los macuna son más distantes debido al peso que tienen las carreras ceremoniales y al temor a ataques chamanísticos, pero quizás también debido a tres siglos de contacto con discursos cristianos que promulgaban unas relaciones jerárquicas entre géneros y la separación de los espacios femeninos y masculinos como una forma de respeto y de "evitar las tentaciones". 
La segunda tiene que ver con las interacciones entre sexo y afecto, que como construcciones históricas y culturales se transforman. Hasta ahora he mostrado cómo estos indígenas hoy consideran que el sexo es más placentero y seguro con el cónyuge, cómo mujeres y hombres tienen sexo por fuera de las uniones maritales, cómo ciertas prácticas "homosexuales" son permitidas entre los jóvenes y cómo tras percibir el sexo como una actividad fisiológica más, subyace un principio de reciprocidad e intercambio que implica tanto la satisfacción de los deseos y necesidades de uno como del otro. Entonces, esta perspectiva, que puede ser catalogada de básica o pragmática, ¿́cómo está siendo transformada por nuestros imaginarios románticos que ligan el sexo con la promesa de una entrega amorosa exclusiva $e$ incondicional entre dos individuos? Y al mismo tiempo ¿́cómo cuestiona nuestro desplazamiento del sexo asociado a la reproducción social al sexo concebido como parte esencial del desarrollo de un sujeto individual?

\section{Referencias bibliográficas}

ÅRHEM, Kaj. Cómo conseguir esposa entre los Makuna. Informes Antropológicos n 3, Bogotá, 1989, pp.15-31.

.S.f. Ecosofía Makuna. En: Correa, F. (ed.), La selva humanizada: ecología alternativa en el trópico húmedo colombiano. Bogotá, Instituto Colombiano de Antropología, Fondo FEN Colombia y Cerec, pp.105-122.

. Ecocosmología y chamanismo en el Amazonas: variaciones sobre un tema. Revista Colombiana de Antropología 37, Bogotá, 2001, pp.268-288.

BELAÚNDE, Luisa Elvira. Viviendo bien: género y fertilidad entre los airopai de la Amazonía peruana. Lima, Centro Amazónico de Antropología y Aplicación Práctica y Banco Central de Reserva del Perú, 2001. 
Sexualidad y afecto entre los macuna y los nükak...

CABRERA, Gabriel; Franky, Carlos y Mahecha, Dany. Los Nukak: nómadas de la Amazonia Colombiana. Bogotá, Unibiblos y Fundación GaiaAmazonas, 1999.

CAYÓn, Luis. En las aguas de Yuruparí: Cosmología y chamanismo makuna. Bogotá, Uniandes, Facultad de Ciencias Sociales, Departamento de Antropología, Ceso, 2002.

. Penso, logo crio. A teoria makuna do mundo. Ph.D. Programa de Pós-graduação em Antropologia Social. Universidade de Brasília, 2010.

FouCAult, Michel. La historia de la sexualidad Tomo 1. Madrid, Siglo XXI, 1977.

FRANKY, Carlos. Territorio y territorialidad indígena. Un estudio de caso entre los tanimuca y el Bajo Apaporis (Amazonia colombiana). Tesis de maestría en Estudios Amazónicos, Universidad Nacional de Colombia, Leticia, 2004.

'Acompañarnos contentos con la familia': Unidad, diferencia y conflicto entre los Nükak. (Amazonia colombiana). Ph.D. dissertation, Wageningen University, 2011.

Gow, Peter. Husband and Wife, Of Mixed Blood: Kinship and History in Peruvian Amazon. Oxford, Oxford University Press, 1991.

Gregor, Thomas. Capts 2, 3 y 4. En: Anxious pleasures. The Sexual Lives of an Amazonian People. Chicago and London, The University of Chicago Press, 1985, pp.10-68.

LONDOÑO, Carlos. Muinane: un proyecto moral a perpetuidad. Medellín, Universidad de Antioquia, 2004.

MAHECHA, Dany. Formación de masá goro 'personas verdaderas'. Pautas de crianza entre los macuna del bajo Apaporis. Tesis de maestría en Estudios Amazónicos, Universidad Nacional de Colombia, Leticia, 2004.

. El nombre en Nikak. En: WeTzELS, L. (ed.) The linguistics of endangered languages. Contributions to morphology and morphosyntax. Utrecht, LOT, 2009, pp.63-93. 
MAHECHA, Dany y FrANKY, Carlos. Recolectando en el cielo. Elementos del manejo nukak del mundo (Amazonia colombiana). En: EPPS, P. y STENZEL, K. (eds.) Interaction among peoples of the Upper Rio Negro Region (Amazonia): Cultural and linguistic perspectives. Rio de Janeiro, Museu Nacional Museu do Índio - Funai, 2013, pp.163-196.

MAHECHA, Dany; Franky, Carlos; GutiÉRREZ, Ruth; y Olmedo MARTínEZ, Luis. Contacto, desplazamiento forzado y Cambios entre los Nükak. En: MAHECHA, D. y FRANKY, C. (eds.) Los Nükak. El último pueblo de tradición nómada contactado oficialmente en Colombia. Copenhague, IWGIA, 2011, pp.10-29.

OVERING, Joanna y PASSES, Alan. Introduction: Conviviality the opening up of Amazonian anthropology. En: OVERING, J. y PASSES, A. (eds.) Anthropology of Love and Anger: The Aesthetics of Conviviality in Native Amazonia. London, New York, Routledge, 2000, pp.1-30.

RIVAL, Laura. Hijos del sol, padres del jaguar: los huaorani de ayer y hoy. Quito, Abya-Yala, 1996.

Androgynous Parents and Guest Children: The Huaorani Couvade. The Journal of the Royal Anthropological Institute 4 (4), Londres, 1998, pp.619-642.

. What kind of sex makes people happy?. En: ASTUTI, R.; PARRY, J. y STAFFORD, C. (Eds.) Questions of anthropology. Festschrift for Maurice Bloch. Oxford, Berg, 2007, pp.167-196.

RUBIN, Gayle. El tráfico de mujeres: notas sobre la "economía política" del sexo. Nueva Antropología, vol. VIII, n 30, México, 1986, pp.95-145.

VILAÇA, Aparecida. Making kin out of others in Amazonia. The Journal of the Royal Anthropological Institute 8 (2), Londres, 2002, pp.347-365.

VIVEIROS DE CASTRO, Eduardo. Perspectivismo e multinaturalismo na América indígena. En: VIVEIROS DE CASTRO, E. A inconstância da alma selvagem e outros ensaios de antropologia. São Paulo, Cosac y Naify, 2002, pp.345-399.

WEEKS, Jeffrey. Invented moralities. Sexual values in an age of uncertainty. Cambridge, Polity Press, 1995. 